Network Working Group

Request for Comments: 2160

Category: Standards Track
H. Alvestrand

UNINETT

January 1998

\title{
Carrying PostScript in X.400 and MIME
}

Status of this Memo

This document specifies an Internet standards track protocol for the Internet community, and requests discussion and suggestions for improvements. Please refer to the current edition of the "Internet Official Protocol Standards" (STD 1) for the standardization state and status of this protocol. Distribution of this memo is unlimited.

Copyright Notice

Copyright (C) The Internet Society (1998). All Rights Reserved.

Table of Contents

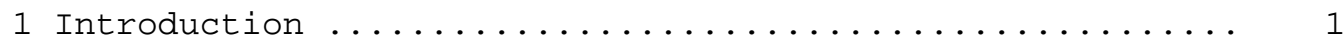

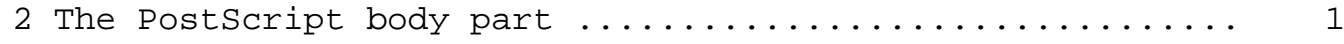

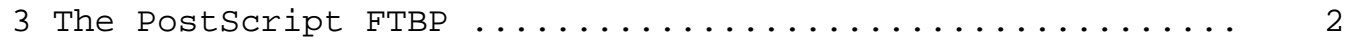

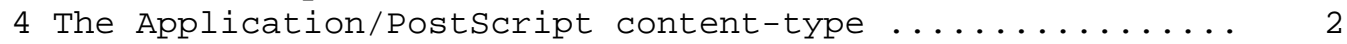

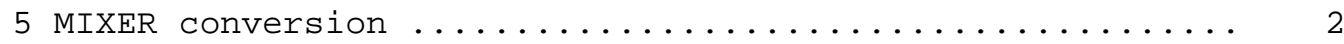

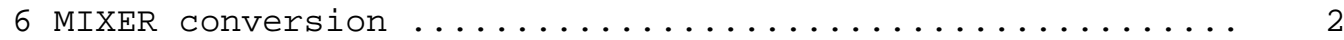

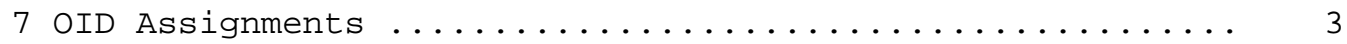

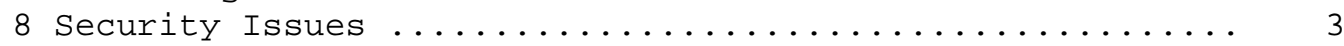

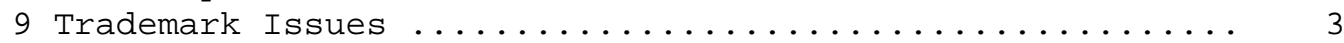

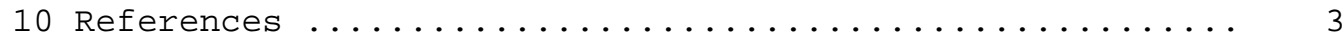

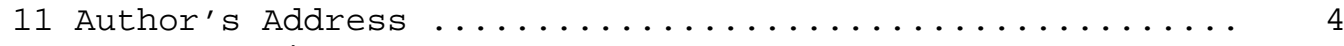

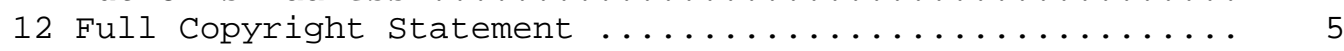

1. Introduction

This document describes methods for carrying Postscript information in the two standard mail systems MIME and X.400, and the conversion between them. It uses the notational conventions of [BODYMAP], and the conversion is further described in [MIXER].

Two ways of carrying Postscript in X.400 are described. One is using the FTAM Body Part, and one uses the Extended Body Part originally described in RFC 1494.

The FTAM method is recommended. 
2. The Postscript body part

Carrying Postscript in X.400 as an Extended Body Part was originally defined in RFC 1494. This specification carries that work forward now that RFC 1494 is obsoleted by [BODYMAP].

The following Extended Body Part is defined for Postscript data streams. It has no parameters.

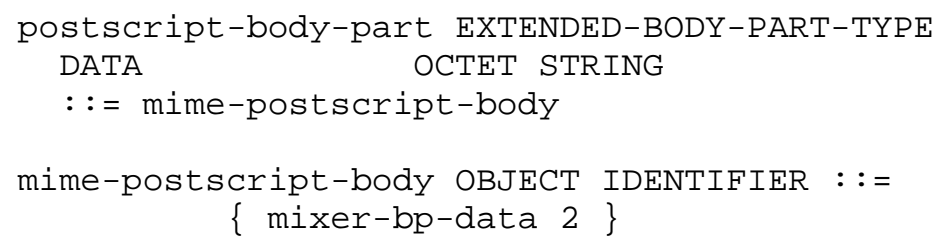

3. The Post Script FTBP

The PostScript FTBP is identified by having the FileTransferparameters.environment.application-reference set to idmime-ftbp-postscript.

The definition is:

id-mime-ftbp-postscript OBJECT IDENTIFIER : := $\{$ mixer-bp-data 6$\}$

4. The Application/PostScript content-type

In MIME, PostScript is carried in the body part

"application/PostScript", which is defined in RFC 1521.

5. MIXER conversion

X.400 Body Part: Extended Body Part, OID mime-postscript-body MIME Content-Type: application/postscript Conversion Type: No conversion

The two representations of PostScript both contain a single stream of octets. This stream of octets can be copied with no problems between the representations. No other data needs to be converted.

6. MIXER conversion

X.400 Body Part: FTBP, OID mime-ftbp-postscript-body MIME ContentType: application/postscript Conversion Type: No conversion 
The two representations of PostScript both contain a single stream of octets. This stream of octets can be copied with no problems between the representations. No other data needs to be converted.

7. OID Assignments

The first OID is also defined in [BODYMAP].

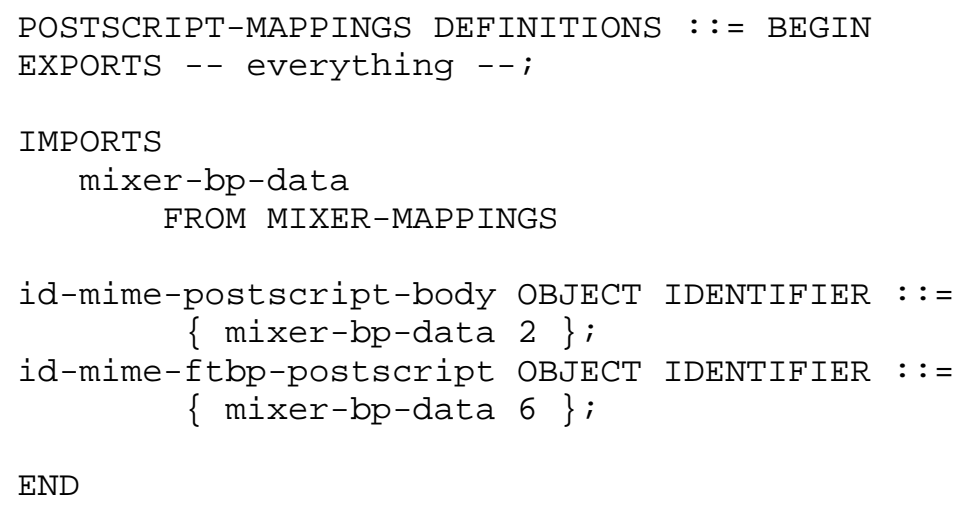

END

8. Security Issues

The issues concerning Post Script and security are well discussed in RFC 2046. No additional security issues are identified by this memo.

9. Trademark Issues

PostScript is a trademark of Adobe Systems, Inc.

10. References

[MIXER]

Kille, S., "MIXER: Mapping between X.400

and RFC 822/MIME", RFC 2156, January 1998.

[BODYMAP ]

Alvestrand, H., "Mapping between X.400 and RFC 822/MIME

Message Bodies", RFC 2157, January 1998. 
11. Author's Address

Harald Tveit Alvestrand UNINETT

Postboks 6883 Elgeseter N-7002 TRONDHEIM

Phone: +47 $73 \quad 5970 \quad 94$

EMail: Harald.T.Alvestrandeuninett.no 


\section{Full Copyright statement}

Copyright (C) The Internet Society (1998). All Rights Reserved.

This document and translations of it may be copied and furnished to others, and derivative works that comment on or otherwise explain it or assist in its implementation may be prepared, copied, published and distributed, in whole or in part, without restriction of any kind, provided that the above copyright notice and this paragraph are included on all such copies and derivative works. However, this document itself may not be modified in any way, such as by removing the copyright notice or references to the Internet society or other Internet organizations, except as needed for the purpose of developing Internet standards in which case the procedures for copyrights defined in the Internet Standards process must be followed, or as required to translate it into languages other than English.

The limited permissions granted above are perpetual and will not be revoked by the Internet society or its successors or assigns.

This document and the information contained herein is provided on an "AS IS" basis and THE INTERNET SOCIETY AND THE INTERNET ENGINEERING TASK FORCE DISCLAIMS ALL WARRANTIES, EXPRESS OR IMPLIED, INCLUDING BUT NOT LIMITED TO ANY WARRANTY THAT THE USE OF THE INFORMATION HEREIN WILL NOT INFRINGE ANY RIGHTS OR ANY IMPLIED WARRANTIES OF MERCHANTABILITY OR FITNESS FOR A PARTICULAR PURPOSE. 